Check for updates

Cite this: DOI: 10.1039/d0fo01148g

\title{
(-)-Epicatechin protects thoracic aortic perivascular adipose tissue from whitening in high-fat fed mice $\dagger$
}

\author{
Ezequiel J. Hid, ${ }^{a, b}$ Laura Fischerman, ${ }^{a, b}$ Barbara Piotrkowski, ${ }^{a, b}$ María C. Litterio, ${ }^{a, b}$ \\ Cesar G. Fraga (iD a,b,c and Monica Galleano iD *a,b
}

\begin{abstract}
High adipose tissue (AT) accumulation in the body increases the risk for many metabolic and chronic diseases. This work investigated the capacity of the flavonoid (-)-epicatechin to prevent undesirable modifications of AT in mice fed a high-fat diet. Studies were focused on thoracic aorta perivascular AT (taPVAT), which is involved in the control of blood vessel tone, among other functions. Male C57BL/6J mice were fed for 15 weeks a high-fat diet with or without added (-)-epicatechin (20 mg per kg body weight per d). In high-fat diet fed mice, (-)-epicatechin supplementation: (i) prevented the expansion of taPVAT, (ii) attenuated the whitening of taPVAT (according to the adipocyte morphology, diameter, and uncouplingprotein 1 (UCP-1) levels) and (iii) blunted the increase in plasma glucose and cholesterol. The observed taPVAT modifications were not associated with alterations in the aorta wall thickness, aorta tumor necrosis factor-alpha (TNF- $\alpha$ ) and NADPH-oxidase 2 (NOX2) expression, and endothelial nitric oxide synthase (eNOS) phosphorylation levels. In summary, our results indicate (-)-epicatechin as a relevant bioactive protecting from the slow and silent development of metabolic and chronic diseases as they are associated with excessive fat intake.
\end{abstract}

Received 4th May 2020

Accepted 11th June 2020

DOI: $10.1039 /$ dOfo01148g

rsc.li/food-function in adult subjects. ${ }^{2,3} \mathrm{~A}$ characteristic that differentiates WAT and BAT is the density of mitochondria in adipocytes, which is lower in WAT than in BAT. Also, the use of energy favors lipid storage in WAT but dissipates as heat in BAT. In addition, a third type of AT exists surrounding blood vessels, i.e. the perivascular (PVAT). This AT displays features of WAT and BAT. ${ }^{4}$ In rodents, PVAT surrounding the abdominal aorta exhibits a WAT-like phenotype, but the PVAT surrounding the thoracic aorta (taPVAT) is more similar to BAT in terms of morphology and functions.

AT pads can interchange their structural, cellular and molecular characteristics in response to both physiological and pathological conditions. ${ }^{5}$ This plasticity can result in positive or negative health effects. Positive examples are the shift from WAT to BAT (browning) after cold exposure or physical activity. In contrast, undesirable changes from BAT to WAT (whitening) occur associated with age and obesity. ${ }^{6}$

Flavonoids are compounds present in edible fruits and vegetables, and increasing evidence supports the benefits of their consumption in human health. ${ }^{7}$ Diets rich in flavonoids could contribute to the browning of WAT. ${ }^{8}$ Particularly, the flavonoid (-)-epicatechin has been associated with positive health effects affecting several disease conditions, including obesity. ${ }^{9-16}$ In terms of mechanisms of disease, (-)-epicatechin supplementation has been shown to mitigate systemic and 
WAT insulin resistance in high-fat fed mice, ${ }^{15}$ in part due to its capacity to inhibit WAT inflammation, endoplasmic reticulum stress and oxidative stress. ${ }^{17,18}$

In this work, we investigated the capacity of (-)-epicatechin to prevent pathological modifications of AT developed in mice fed a high-fat diet. We observed that (-)-epicatechin supplementation attenuated the high-fat induced whitening of taPVAT.

\section{Materials and methods}

\section{Materials}

Primary antibodies for inducible nitric oxide synthase (iNOS) (\#649), endothelial nitric oxide synthase (eNOS) (\#654), interleukin-6 (IL-6) (\#1265), tumor necrosis factor (TNF- $\alpha$ ) (\#52746), NOX2 catalytic subunit gp91(\#5827), and $\beta$-actin (\#47778), and secondary antibodies, mouse anti-rabbit IgG-HRP (\#2357), goat anti-mouse IgG-HRP (\#2005), and rabbit anti-goat IgG-HRP (\#2768), were from Santa Cruz Biotechnology (Santa Cruz, CA, USA). The primary antibody for phospho-eNOS (Ser 1177) (p-eNOS) (\#9570) was from Cell Signaling Technology (Danvers, MA, USA). The primary antibodies for uncouplingprotein 1 (UCP-1) (\#U6382) and (-)-epicatechin were from Sigma Aldrich (St Louis, MO, USA).

\section{Animals, diets and experimental design}

All procedures were in agreement with standards for the care of laboratory animals as outlined in the National Institutes of Health Guide for the Care and Use of Laboratory Animals (NIH Pub. no. 85-23, Revised 1996) and were approved by the Institutional Committee for the Care and Use of Laboratory Animals, School of Pharmacy and Biochemistry, University of Buenos Aires, Argentina (CUDAP: EXP-UBA: 75405/16).

Male C57BL/6J mice were housed under conditions of controlled temperature $\left(21-25^{\circ} \mathrm{C}\right)$ and humidity, with a $12 \mathrm{~h}$ light/ dark cycle. Mice (8 per group) weighing $20 \pm 2 \mathrm{~g}$ were randomly divided into the following groups depending on the diet: (i) control group (C), receiving a control diet (10\% of total calories from lard fat); (ii) control-epicatechin group (CE), receiving the control diet supplemented with (-)-epicatechin $(20 \mathrm{mg}$ per $\mathrm{kg}$ body weight per d); (iii) high-fat group (HF) receiving a highfat diet (60\% of total calories from lard fat); and (iv) high-fatepicatechin group (HFE), receiving the high-fat diet supplemented with (-)-epicatechin (20 $\mathrm{mg}$ per $\mathrm{kg}$ body weight per d). ${ }^{18,19}$ The amount of (-)-epicatechin provided to mice is equivalent to $200 \mathrm{mg} \mathrm{d}^{-1}$ for a $70 \mathrm{~kg}$ human, quantity attainable through the optimization of fruit and vegetable intake and/or pharmacological strategies. ${ }^{10,11}$ The control and highfat diet composition is shown in the ESI. $\dagger$ Pellets were prepared by adding the fat lard or/and (-)-epicatechin once every two weeks, to adjust the amount of (-)-epicatechin according to food consumption and animal weight. Dry pellets were stored at $4{ }^{\circ} \mathrm{C}$. Food intake consumption and body weight were recorded weekly. After 15 weeks of the respective treatments, mice were weighed, and euthanized in a $\mathrm{CO}_{2}$ chamber. Blood was collected from the abdominal aorta into heparinized tubes and plasma was obtained after centrifugation at $600 \mathrm{~g}$ for $15 \mathrm{~min}$ at $4{ }^{\circ} \mathrm{C}$. Blood plasma samples were frozen at $-80^{\circ} \mathrm{C}$. The clean aorta and the aorta surrounded by taPVAT, eWAT, mWAT, rWAT, and pWAT were excised immediately and either processed for histology or flash frozen in liquid $\mathrm{N}_{2}$ for further analyses.

\section{Biochemical determinations}

Glucose, total cholesterol, and triglycerides in plasma were measured using a Cobas C-501 autoanalyzer (Roche Diagnostics, Mannheim, Germany).

\section{Western blotting analysis}

eWAT and mWAT were homogenized (proportion $1: 3, \mathrm{w}: \mathrm{v}$ ) in lysis buffer (150 mM NaCl, $50 \mathrm{mM}$ Trizma-HCl, 1\% (v/v) NP-40, $\mathrm{pH}$ 8.0) in the presence of protease and phosphatase inhibitors, and centrifuged at $600 \mathrm{~g}$ for $10 \mathrm{~min}$ at $4{ }^{\circ} \mathrm{C}$. The supernatant was collected and considered as total homogenates. Total homogenates were added to a $2 \times$ solution of Laemmli buffer and heated at $95{ }^{\circ} \mathrm{C}$ for $5 \mathrm{~min}$. Sample aliquots containing $40 \mu \mathrm{g}$ of protein were separated by reducing $10 \%(\mathrm{w} / \mathrm{v})$ polyacrylamide gel electrophoresis, and electroblotted onto polyvinylidenedifluoride membranes. Colored molecular weight standards (GE Healthcare, Piscataway, NJ, USA) were run simultaneously. Membranes were blotted for $2 \mathrm{~h}$ in $5 \%(\mathrm{w} / \mathrm{v})$ nonfat milk and incubated overnight in the presence of the corresponding primary antibody (1:1000 dilution in PBS). After a subsequent incubation for $90 \mathrm{~min}$ at room temperature in the presence of the corresponding HRP-conjugated secondary antibody ( $1: 5000$ dilution in PBS), complexes were visualized by chemiluminescence. Films were scanned and the densitometry analysis was performed using Image J (National Institute of Health, Bethesda, Maryland, USA). Proteins were normalized to the $\beta$-actin content. The protein content of total homogenates was measured by the Lowry method. ${ }^{20}$

\section{Histological and immunochemical analysis of AT and aorta}

For each animal, a portion of thoracic aorta with surrounding taPVAT and a portion of eWAT and mWAT were separated, fixed in phosphate-buffered $10 \%(\mathrm{v} / \mathrm{v})$ formaldehyde $(\mathrm{pH} 7.2)$ and embedded in paraffin. Three $\mu \mathrm{m}$ sections were cut and stained with hematoxylin-eosin or Trichrome Masson's stains. Histological evaluations were performed using a light microscope Nikon E400 (Nikon Instrument Group, Melville, NY, USA). To evaluate aorta morphometry, the wall media thickness and lumen diameter were measured. To establish expansion of taPVAT, the area of taPVAT was relativized to the aortic wall media thickness. Immunohistochemistry of taPVAT was evaluated with antibodies against UCP-1 (1:100 in PBS). Immunostaining was quantified as the percentage of positive staining per area from 20 random images viewed at $\times 400$ magnification. Measurements were performed using Image-Pro Plus version 4.5 for Windows (Media Cybernetics, LP, Silver Spring, MD, USA). 


\section{Statistical analysis}

Data from food intake, energy intake, body weight, biochemical parameters, and western blotting analysis were analyzed by one-way ANOVA followed by Tukey-Kramer's post-hoc test using StatView5.0 (SAS Institute, Cary, NC, USA). Histological and immunohistochemical data were analyzed by the nonparametric Kruskal-Wallis test followed by Dunn's post-test using GraphPad Prism 5.01 (GraphPad Software, Inc. San Diego, CA, USA). All data are presented as mean \pm standard error of the mean (SEM) with significance set at $p<0.05$.

\section{Results}

Food consumption, energy intake, and body weight during the treatment period are shown in Fig. 1. Daily food consumption did not change significantly because of the different treatments. HF and HFE consumed lower amounts of food compared to $\mathrm{C}$ and $\mathrm{CE}$ (Fig. 1A), with differences among groups already being observed in the first week under treatment. The energy intake was calculated considering the food consumption and the caloric value of control and high fat diets, yielding similar caloric intakes among the four groups (Fig. 1B). Body weight increases were similar for the four experimental groups during the first 4 weeks, and were higher for the groups receiving the high-fat diet during the remaining treat- ment period (Fig. 1C). The final body weight in HF and HFE was significantly higher than that observed in $\mathrm{C}$ and $\mathrm{CE}$ (Fig. 1D). Body weight gain was independent of the presence of (-)-epicatechin in the diet.

Glycemia and blood lipid parameters were determined as indexes of systemic cardiometabolic responses to the diets (Table 1). Glycemia was significantly higher in HF $(p<0.05)$ as compared to C, CE and HFE. Total plasma cholesterol was significantly higher in $\mathrm{HF}$ as compared to $\mathrm{C}$ and $\mathrm{CE}$; no difference was found for total cholesterol between HFE and, both $\mathrm{C}$ and CE. Triglyceride levels showed no differences among the groups.

The relative mass of eWAT, mWAT, rWAT, and pWAT depots was significantly higher in HF and HFE compared to C and CE

Table 1 Effect of (-)-epicatechin on metabolic parameters in high-fat fed mice

\begin{tabular}{lllll}
\hline Variable $^{a}$ & $\mathrm{CE}$ & $\mathrm{C}$ & $\mathrm{HF}$ & $\mathrm{HFE}$ \\
\left.\hline${\text { Glucose }\left(\mathrm{mg} \mathrm{dl}^{-1}\right)}_{\text {Total cholesterol }\left(\mathrm{mg} \mathrm{dl}^{-1}\right)}\right)$ & $100 \pm 33$ & $275 \pm 40$ & $399 \pm 29^{\#}$ & $307 \pm 12$ \\
Triglycerides $(\mathrm{mg} \mathrm{dl}$ & $99 \pm 8$ & $\left.142 \pm 13^{*}\right)$ & $119 \pm 8$ \\
& $35 \pm 3$ & $47 \pm 8$ & $35 \pm 6$ & $51 \pm 4$
\end{tabular}

${ }^{a}$ Variables were measured in plasma under non-fasting conditions. Results are expressed as means $\pm \operatorname{SEM}(n=8) .{ }^{*} p<0.05 v s$. all other groups. $^{*} p<0.05 v s$. C.
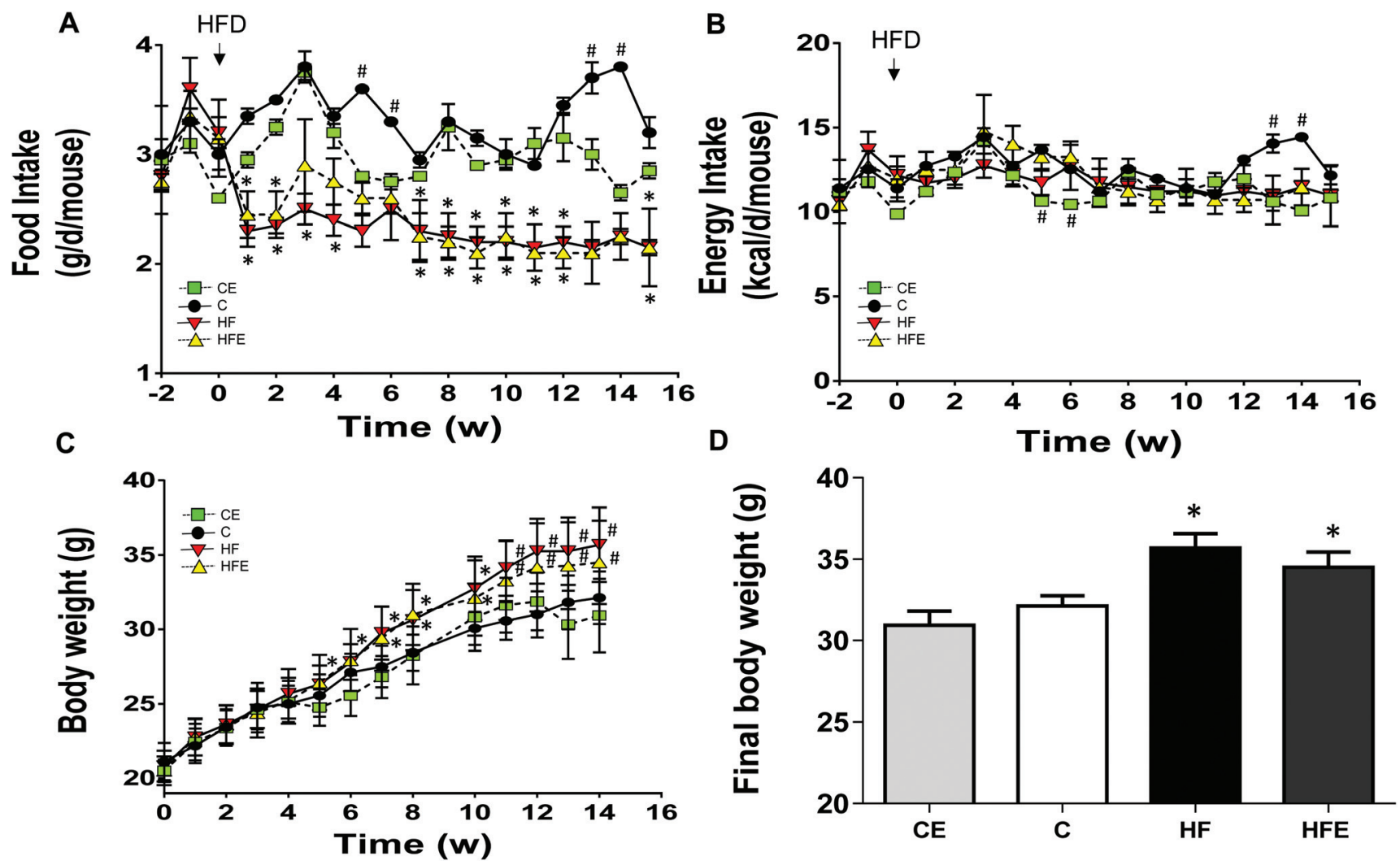

Fig. 1 Food and energy intake and body weight. Daily food intake (A), daily energy intake (B), body weight (C), and final body weight (D) from CE, C, $\mathrm{HF}$ and HFE. Results are expressed as means \pm SEM $(n=8) .{ }^{*} p<0.05$ vs. C and CE. ${ }^{\#} p<0.05$ vs. all other groups. 
(Fig. 2). This resulted in an intra-abdominal adiposity (sum of the four fat pads relative to the body weight) of $84 \pm 6$ and $82 \pm$ $5 \mathrm{mg} \mathrm{g}^{-1}$ for HF and HFE, respectively, that has no significant difference between them, but higher than the values reported for $\mathrm{C}$ and $\mathrm{CE}\left(52 \pm 5\right.$ and $\left.63 \pm 5 \mathrm{mg} \mathrm{g}^{-1}, p<0.05\right)$.

Fig. 3 and 4 show the effects of the high-fat diet and of (-)-epicatechin supplementation on several characteristics of WAT pads. Representative images of hematoxylin-eosin stained eWAT and mWAt are shown in Fig. 3A and 4A, respectively. For eWAT, the analysis of adipocyte size distribution according to its diameter (Fig. 3B) showed that: (i) the percentage of medium $(51-75 \mu \mathrm{m})$ and large adipocytes $(76-100 \mu \mathrm{m})$ was similar for the four experimental groups; (ii) the percentage of small adipocytes $(25-50 \mu \mathrm{m})$ was lower in HF compared with $\mathrm{C}, \mathrm{CE}$ and HFE; and (iii) the percentage of very large adipocytes $(>100 \mu \mathrm{m})$ was higher in $\mathrm{HF}$ and $\mathrm{HFE}$ than for $\mathrm{C}$ and CE. The expression of the pro-inflammatory molecules, IL-6 and iNOS, was evaluated by western blotting. In eWAT, IL-6 expression was similar in $\mathrm{C}, \mathrm{CE}$ and $\mathrm{HF}$, and significantly lower $(\approx 46 \%$ respect to $\mathrm{HF}, p<0.05)$ in $\mathrm{HFE}$ (Fig. 3C). Meanwhile, no significant differences were found in iNOS expression among the four experimental groups (Fig. 3D).
Similar results were observed in mWAT. The adipocyte size distribution results showed that: (i) the percentage of medium (51-75 $\mu \mathrm{m})$ and large adipocytes $(76-100 \mu \mathrm{m})$ was similar in the four experimental groups; (ii) the percentage of small adipocytes $(25-50 \mu \mathrm{m})$ was lower in HF compared with $\mathrm{C}, \mathrm{CE}$ and $\mathrm{HFE}$; and (iii) the percentage of very large adipocytes $(>100 \mu \mathrm{m})$ was higher in $\mathrm{HF}$ and $\mathrm{HFE}$ than in $\mathrm{C}$ and $\mathrm{CE}$ (Fig. 4B). IL-6 expression was similar in C, CE and HF, and significantly lower $(\approx 40 \%$ compared to $\mathrm{HF}, p<0.05)$ in $\mathrm{HFE}$ (Fig. 4C). Meanwhile, no significant differences were found in iNOS expression among the four experimental groups (Fig. 4D).

Morphometric characteristics of taPVAT are shown in Fig. 5A. The expansion of taPVAT was estimated through the ratio of taPVAT area/media thickness of the thoracic aorta. This ratio was significantly higher in $\mathrm{HF}(75 \%, p<0.05)$ compared to C, CE, and HFE (Fig. 5B). Histological characterization of adipocytes showed clear differences between taPVAT characteristics in $\mathrm{C}$ and $\mathrm{CE}$ with respect to HF (Fig. 6A and B). Most of the taPVAT in $\mathrm{C}$ and CE showed a BAT-like appearance (round nuclei, and small and multilocular lipid droplets), and dispersed WAT-like adipocytes (flattened non-central nuclei, and
A

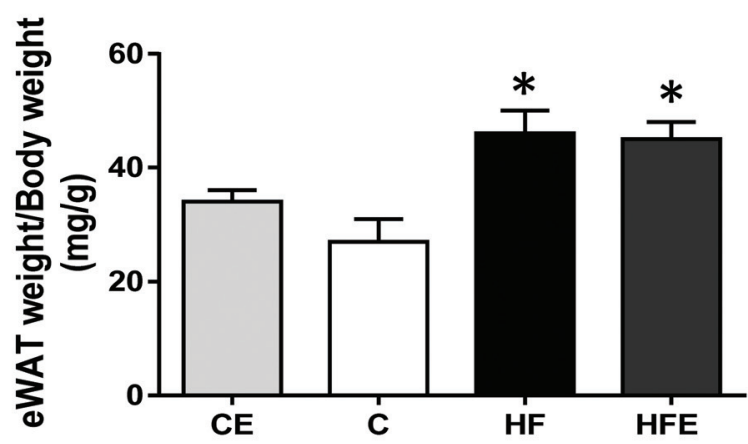

C

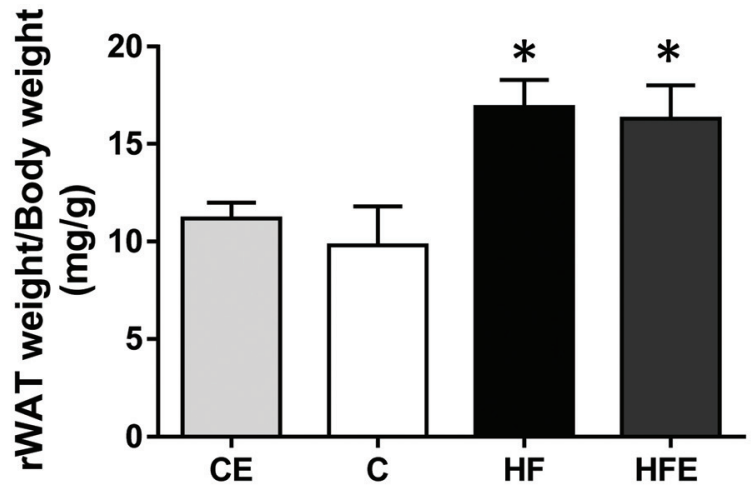

B

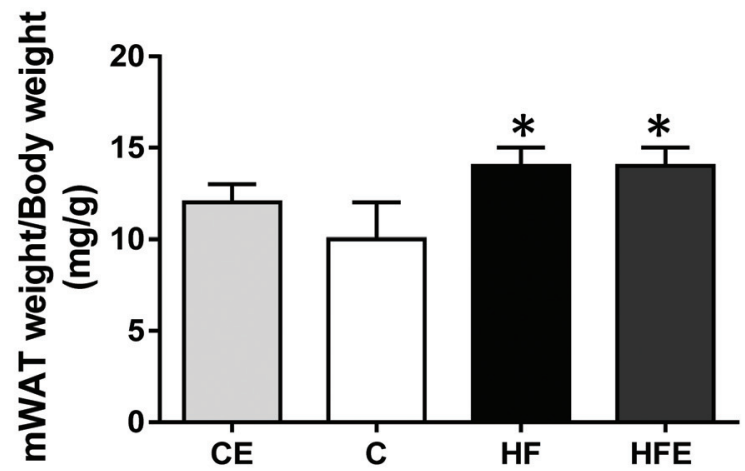

D

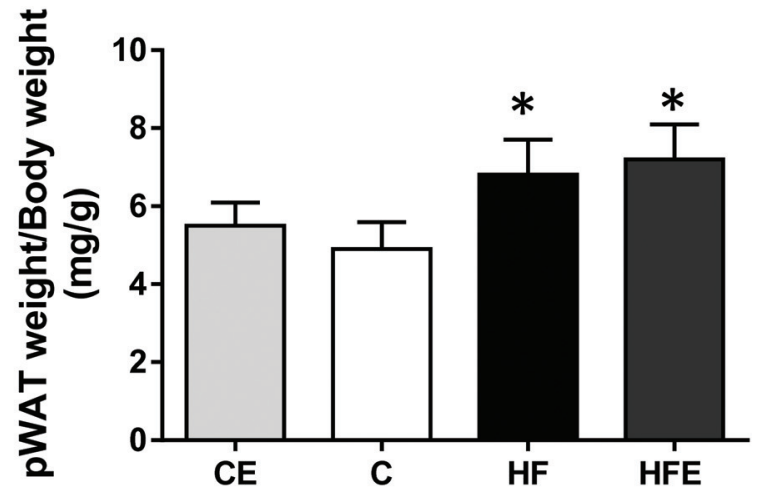

Fig. 2 Relative WAT pad weight. Epididymal (eWAT) (A), mesenteric (mWAT) (B), retroperitoneal (rWAT) (C), and perirenal ( $p W A T)$ (D) from CE, C, HF and HFE. Results are expressed as means \pm SEM $(n=8) .{ }^{*} p<0.05$ vs. C and CE. 
A

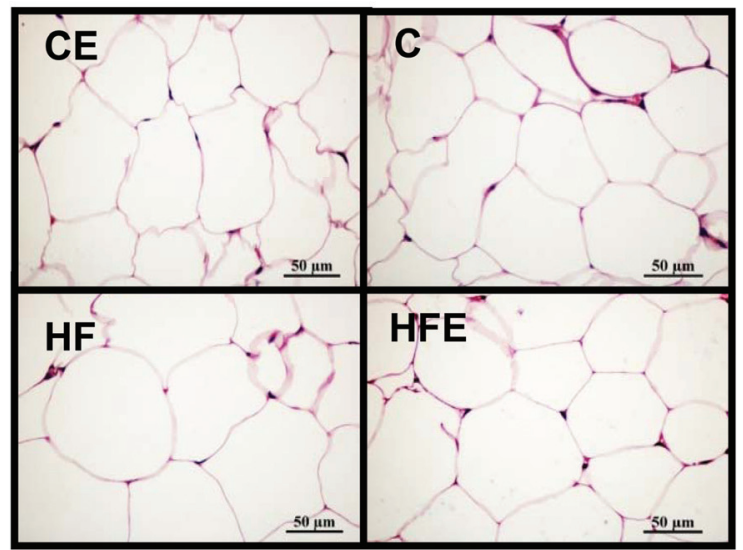

C
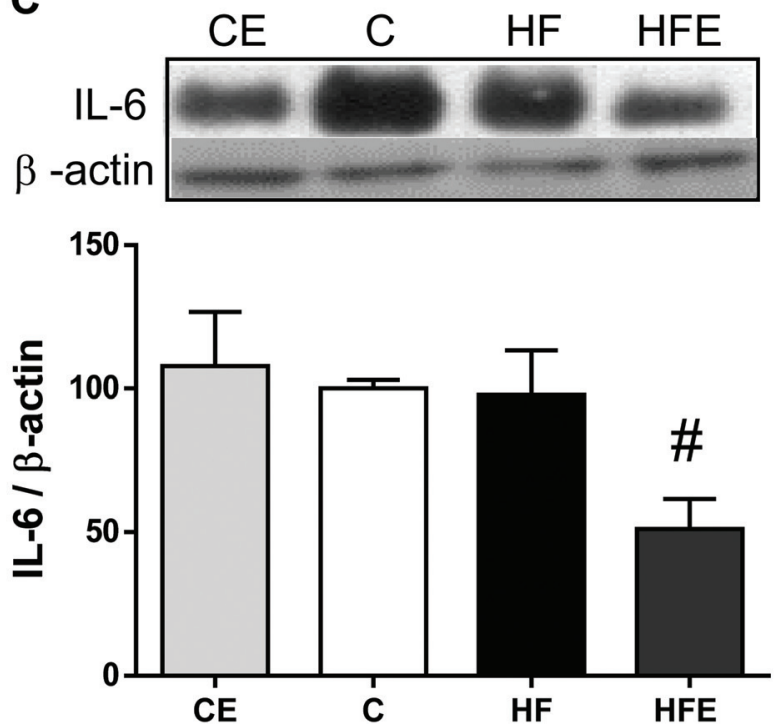

B

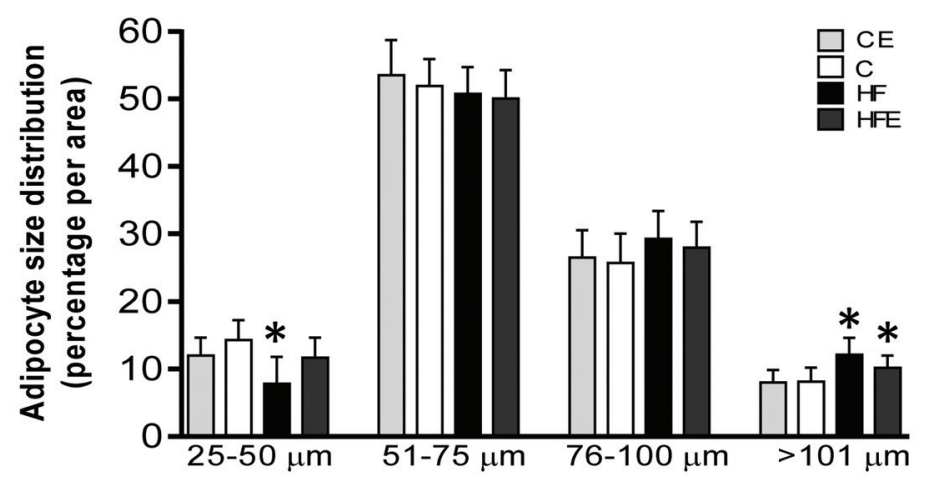

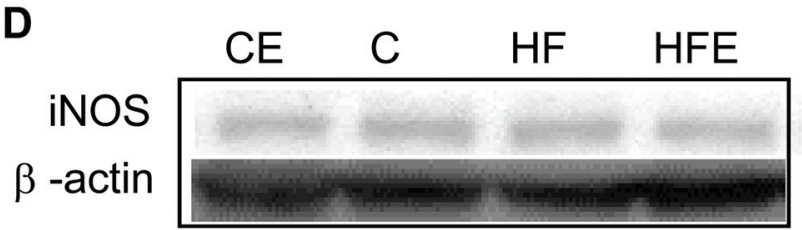

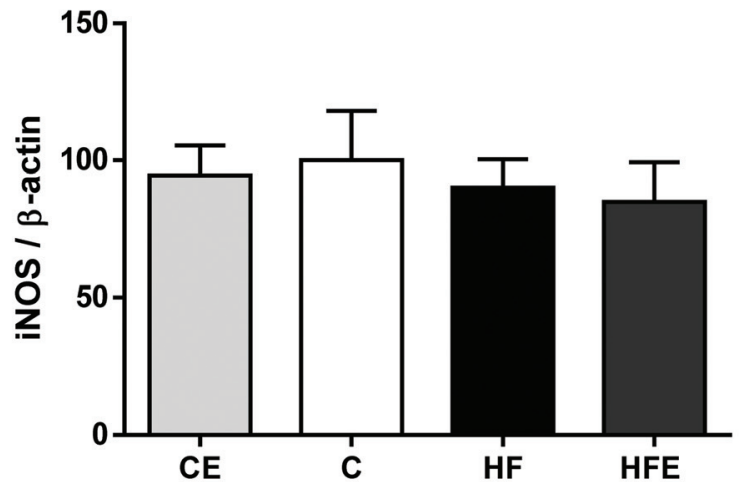

Fig. 3 Cell size distribution and expression of pro-inflammatory molecules in eWAT. eWAT representative images after hematoxylin-eosin staining (A), quantification of eWAT adipocyte size distribution (B), and IL-6 (C) and iNOS (D) expression in eWAT from CE, C, HF and HFE obtained by western blotting. $\beta$-Actin was used as the loading control. Results are expressed as means \pm SEM. For statistics details see the statistical analysis section. ${ }^{*} p<$ 0.05 vs. $C$ and $C E .{ }^{*} p<0.05$ vs. all other groups.

big lipid droplets). In HF there was a clear inversion in the proportion of adipocyte phenotypes from BAT to WAT; meanwhile HFE showed an intermediate phenotype, closer to $\mathrm{C}$ and CE. Quantification of the adipocyte size showed that the diameter was significantly lower in HFE compared to HF $(29 \%, p<0.05)$ (Fig. 6B). Additional confirmation of the BAT characteristics of the taPVAT was obtained by UCP-1 staining. The presence of UCP-1 showed a similar pattern/distribution to that observed for the adipocyte size (Fig. 6A and C). Quantification of UCP-1 staining shows that while about $68 \%$ of staining was observed in $\mathrm{C}$ and $\mathrm{CE}$, only $16 \%$ was observed in $\mathrm{HF}(p<0.05)$. In $\mathrm{HFE}$, the staining was $48 \%$, being significantly higher than that in
HF $(p<0.05)$ and lower than that in C and CE $(p<0.05)$ (Fig. 6D).

The physiological actions of taPVAT result in vascular remodeling and function of the aorta. The four experimental groups showed a similar aorta wall thickness relative to the lumen diameter (Fig. 7A), suggesting the absence of vascular smooth muscle cell proliferation. In addition, no significant changes were observed in the expression of an inflammatory marker in the aorta, such as TNF- $\alpha$ (Fig. 6B) as well as in determinants of nitric oxide bioavailability: (i) the expression of gp91, the catalytic subunit of NOX2 (Fig. 6B and C); and (ii) the phosphorylation of eNOS ( $\mathrm{p}$-eNOS/eNOS). 
A

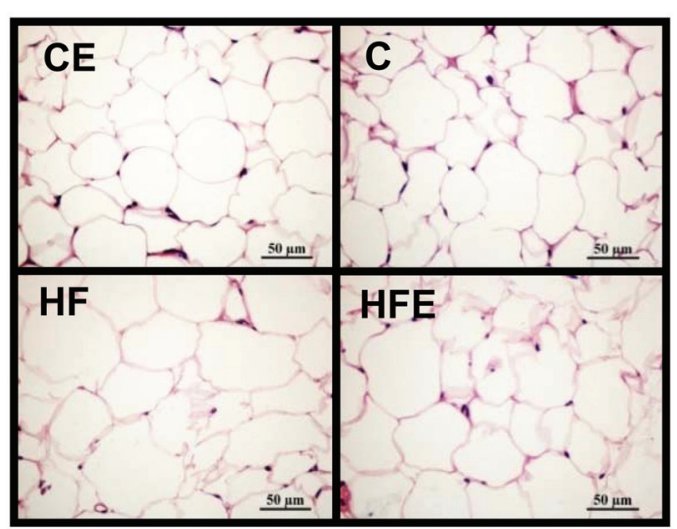

C
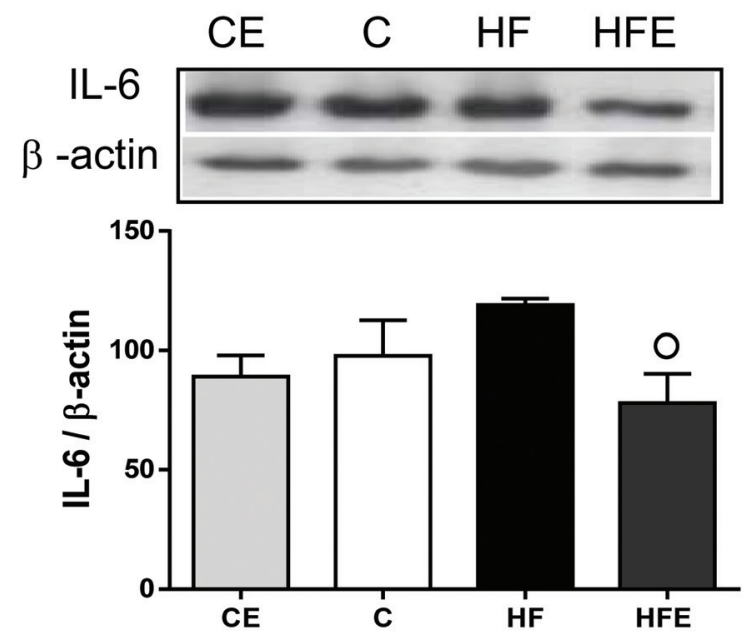

B

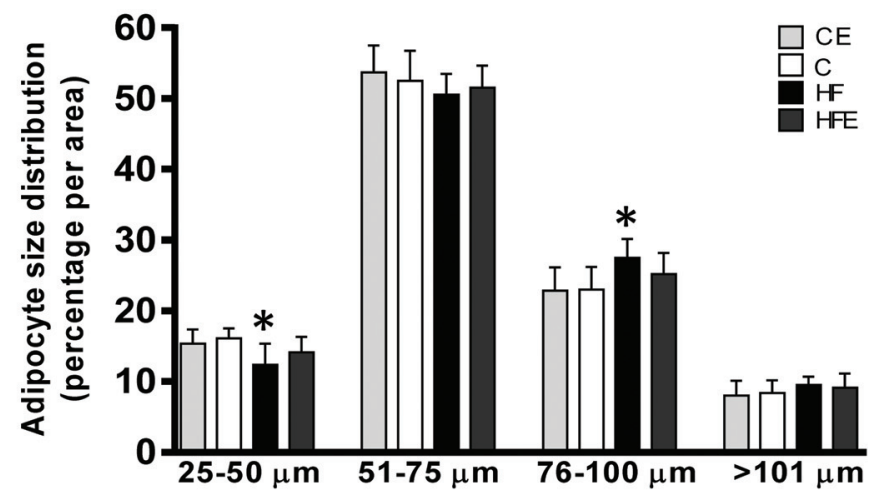

D

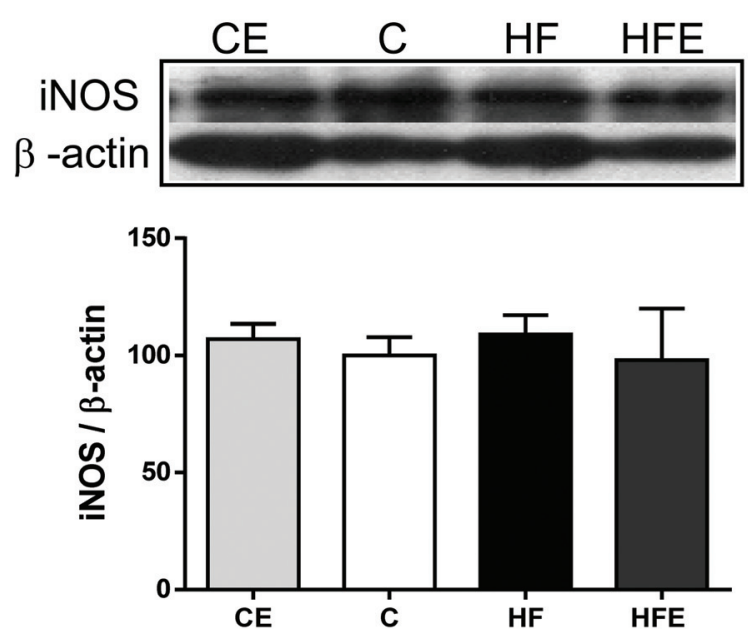

Fig. 4 Cell size distribution and expression of pro-inflammatory molecules in mWAT. mWAT representative images of hematoxylin-eosin staining (A), quantification of mWAT adipocyte size distribution (B), and IL-6 (C) and iNOS (D) expression in mWAT from CE, C, HF and HFE obtained by western blotting. $\beta$-Actin was used as the loading control. Results are expressed as means \pm SEM. For statistics details see the statistical analysis section. ${ }^{*} p<0.05$ vs. C and CE. ${ }^{\circ} p<0.05$ vs. HF.

\section{Discussion}

High AT accumulation in the body increases the risk for many metabolic and chronic diseases. The potential management through bioactives of undesirable changes that accompany the consumption of high calorie diets and AT expansion can have a major impact on health. This work investigated the capacity of (-)-epicatechin to prevent adverse modifications of the AT in mice fed a high-fat diet. A major finding was that (-)-epicatechin supplementation attenuated the whitening of taPVAT, i.e. enlarged adipocytes and lower UCP-1 levels, induced by the high-fat diet. In parallel, the increases in plasma glucose and cholesterol associated with high-fat diet consumption were blunted by (-)-epicatechin.
Experiments were carried out in a $\mathrm{C} 57 \mathrm{BL} / 6 \mathrm{~J}$ sub-strain in which the high-fat diet did not lead to overt obesity. Thus, mice fed the high-fat diet showed a body weight $13 \%$ higher than mice fed the control diet, which is a moderate response compared to other data reported for the same strain, food and time of treatments, i.e. $35-45 \% .{ }^{15,17,18,21}$ This modest increase in weight gain allowed us to analyze the effects of (-)-epicatechin in an early stage of high-fat diet-induced dysmetabolism. The increase in weight in HF and HFE despite the similar caloric intake compared to $\mathrm{C}$ and $\mathrm{CE}$ could be explained by the fact that fat is energetically more efficient than carbohydrates and proteins to promote greater positive energy balance and fat accumulation..$^{22,23}$

In mice consuming the high-fat diet, both body weight and intra-abdominal adiposity were not affected by (-)-epicatechin 

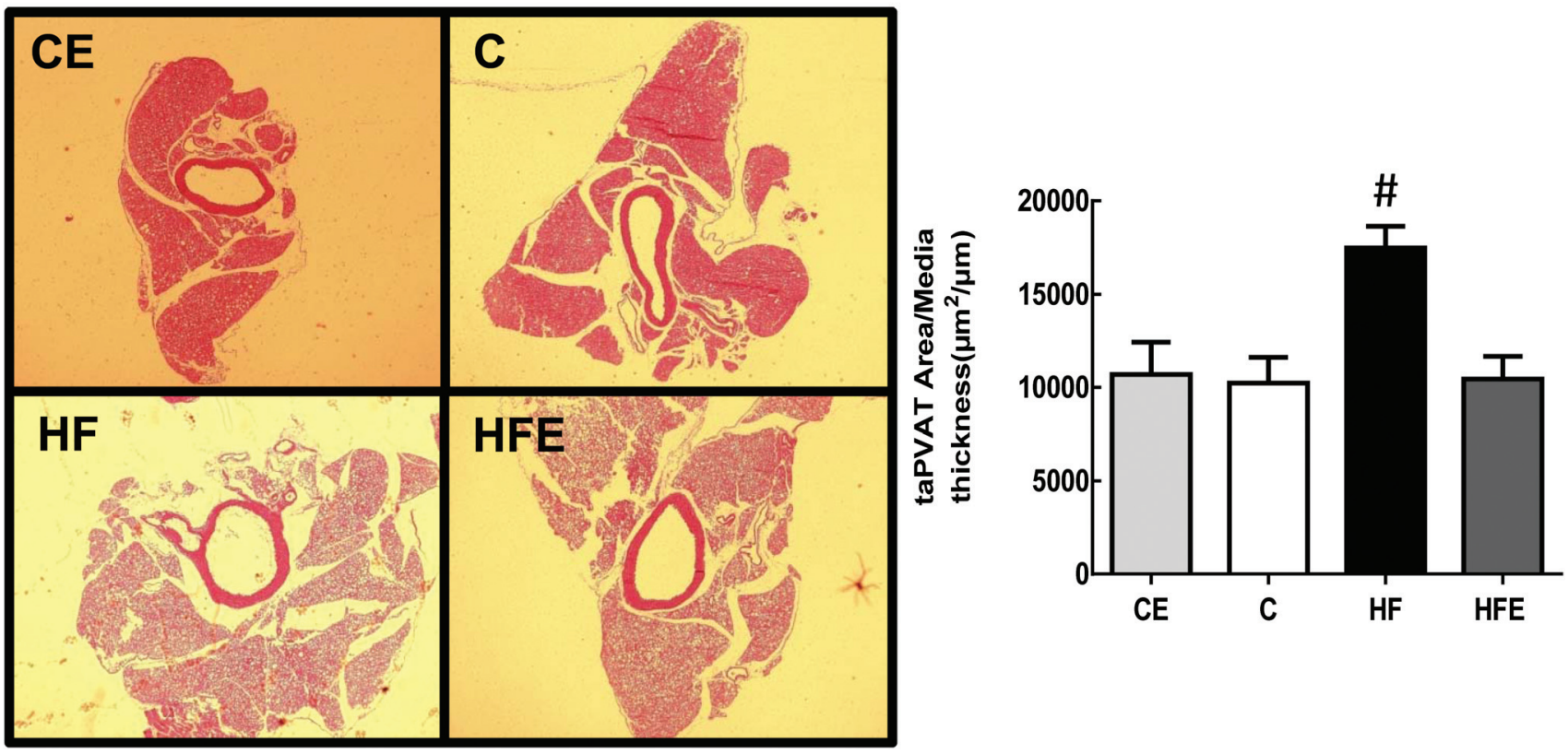

Fig. 5 taPVAT expansion. Transversal sections of the thoracic aorta and taPVAT stained with hematoxylin-eosin (4X) (A), and ratio area of taPVAT/ media layer thickness of the aorta (B) from CE, C, HF and HFE. Results are expressed as means \pm SEM $(n=4)$. ${ }^{*} p<0.05$ vs. all other groups.

supplementation, while increases in glycemia and plasma cholesterol were partially or totally prevented. Mitigation of high-fat induced alterations in plasma glucose and dyslipidemia was previously reported to be associated with (-)-epicatechin intake. ${ }^{15,17,24,25}$ These results support the protective action of (-)-epicatechin in diet-induced metabolic disorders, even in the absence of extreme changes in body weight and/or fat accumulation.

Increasing evidence suggests that the pathogenesis of obesity is to a large extent related to both a pathological expansion of WAT pads and systemic inflammation. ${ }^{26}$ AT expansion can occur through hyperplasia (increased number of cells) and/or hypertrophy (increased cell size). ${ }^{27}$ WAT hypertrophy is characterized by the presence of large and very large adipocytes $^{28}$ with a pro-inflammatory profile. ${ }^{29-31}$ In the present work, the adipocyte size distribution was similar for high-fat fed and control fed mice, suggesting that the predominant expansion mechanism was hyperplasia. In agreement with this, both eWAT and mWAT pads in high-fat fed mice did not show a pro-inflammatory condition as determined by adipocyte levels of IL-6 and iNOS. Interestingly, the minimal changes manifested in the percentage of small and large/very large adipocytes in HF were not present when (-)-epicatechin was supplemented in the diet. These results are in agreement with previous reports showing systemic and local anti-inflammatory effects of (-)-epicatechin ${ }^{32,33}$ and specifically in WAT pads modified by a high-fat diet. ${ }^{17,24}$ Similar effects of (-)-epicatechin were observed even in the offspring of female mice fed a high-fat diet. ${ }^{34}$
PVAT secretes vasocontractile and vasorelaxant factors, involved in the control of blood vessel tone, ${ }^{35}$ as well as a set of molecules with anti-atherogenic and anti-inflammatory functions. ${ }^{36-41}$ In the case of taPVAT, its BAT-like phenotype is crucial for the maintenance of the normal structure and function of the thoracic aorta segment; meanwhile its whitening would be deleterious. This study shows that taPVAT was drastically transformed by the consumption of a high-fat diet, acquiring the features of WAT, while the WAT pads showed a mild expansion, due to adipocyte hyperplasia, without a substantial pro-inflammatory condition. Therefore, the taPVAT whitening emerges as an early hallmark of alterations induced by a high-fat diet. (-)-Epicatechin supplementation was associated with the absence of taPVAT expansion and the attenuation of its transformation into WAT-type adipocytes, as evidenced by adipocyte morphology and UCP-1 expression. Accordingly, (-)-epicatechin has shown effects as a WAT browning agent in experimental models of diet-induced obesity and in adipocytes in culture. In rats fed a high-fat diet, the administration of (-)-epicatechin promoted an increase in the abdominal WAT expression of UCP-1 and deionidase-2, both normally expressed in BAT adipocytes. ${ }^{42}$ More recently, in a similar mouse model, (-)-epicatechin showed a WAT browning effect evidenced by the increased expression of key BATproteins, including UCP- $1 .^{43}$ The modulatory effects of (-)-epicatechin reversing/attenuating the taPVAT whitening could be explained by its capacity to mitigate the down-regulation of BAT of the peroxisome proliferator-activated recep- 
A

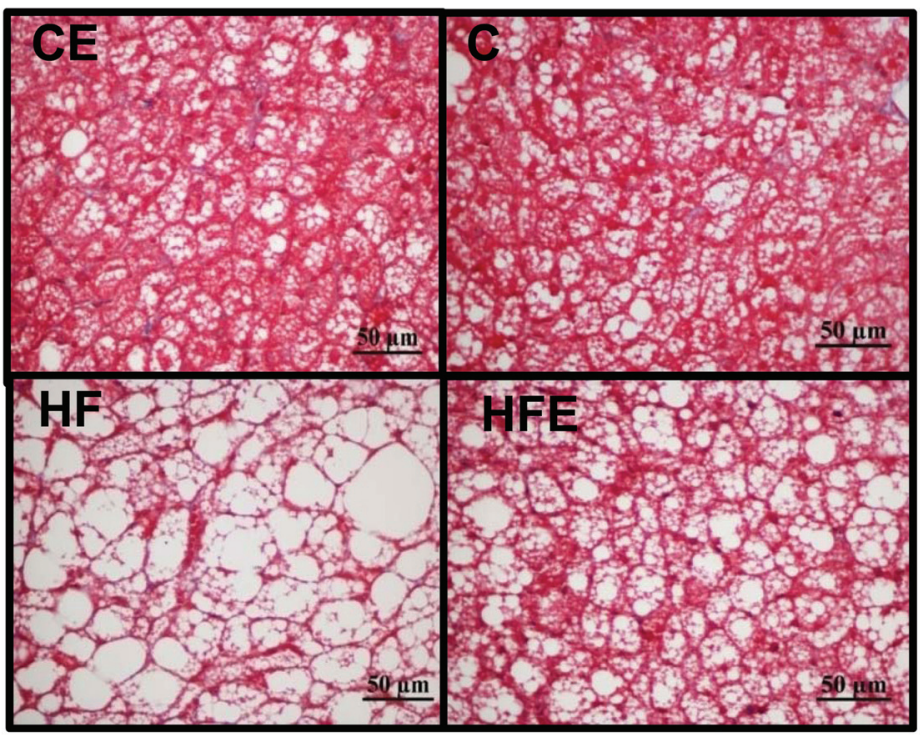

C

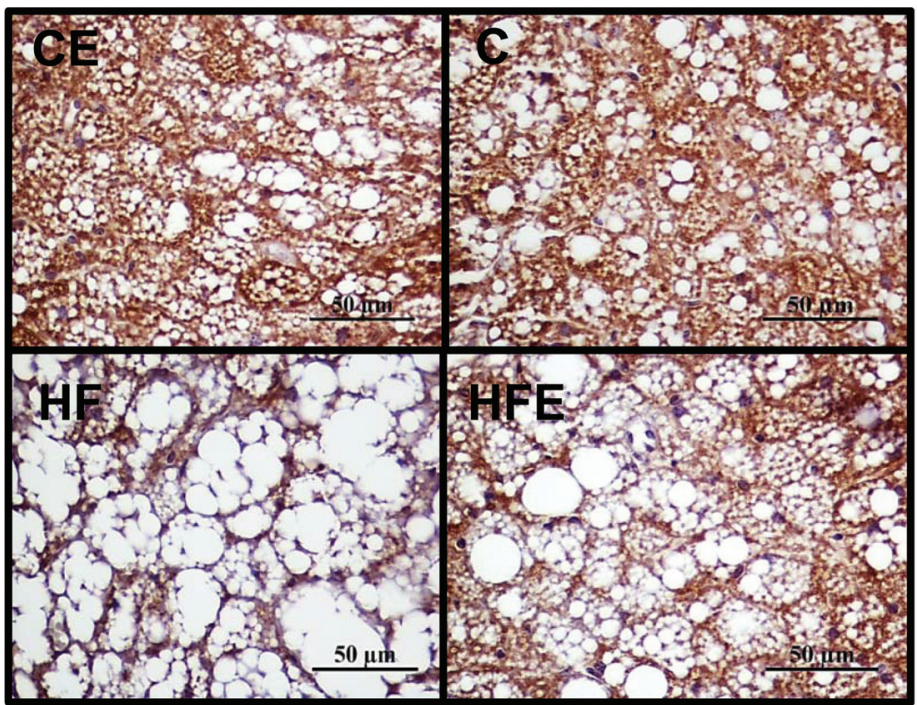

B
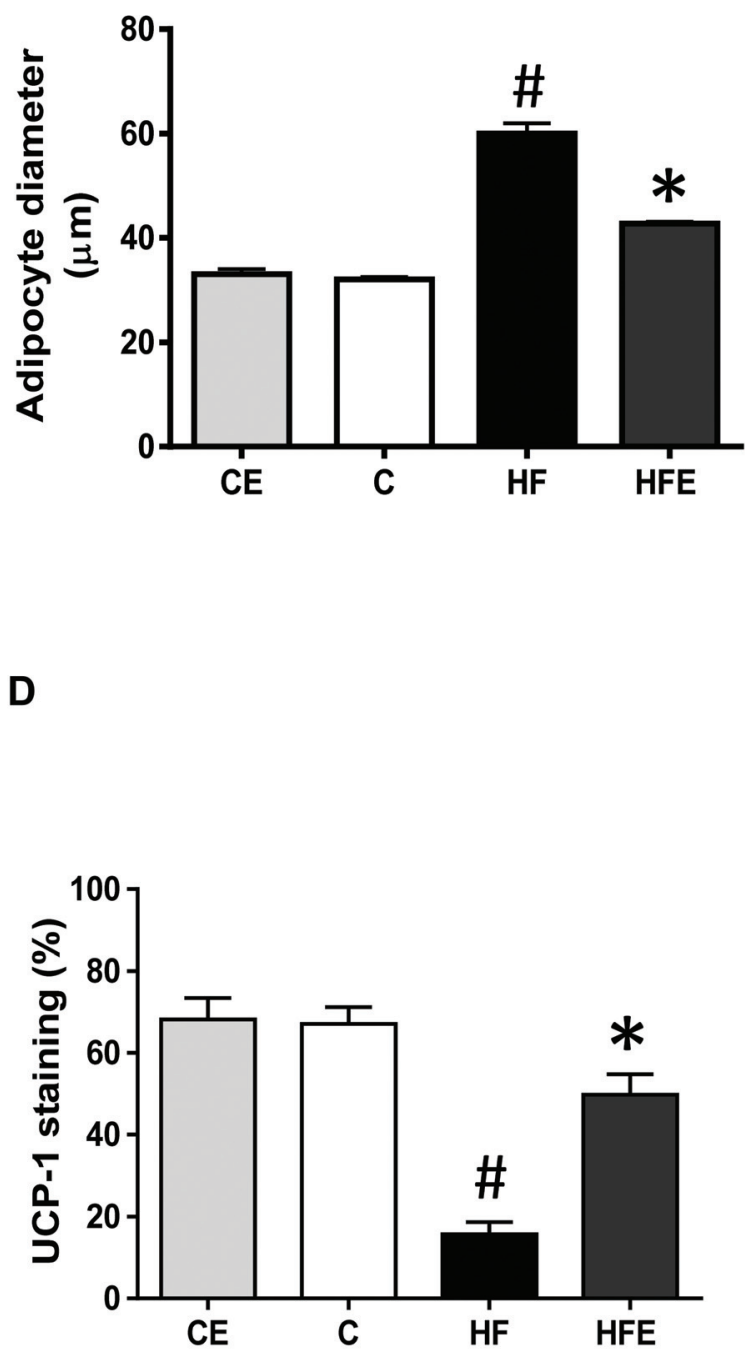

Fig. 6 Cell size and UCP-1 immnunostaining in taPVAT. Representative images (A), and adipocyte diameter (B) of taPVAT stained with Masson's Trichrome (40x), representative images (C), and quantification (D) of UCP-1 staining in taPVAT from CE, C, HF and HFE evaluated with immunohistochemistry. Results are expressed as means \pm SEM $(n=4) .{ }^{*} p<0.05$ vs. C and CE. ${ }^{\#} p<0.05$ vs. all other groups.

tor $\gamma(\operatorname{PPAR} \gamma)$ and other proteins involved in mitochondrial biogenesis (PPAR $\gamma$ coactivador-1 $\alpha$-PGC-1 $\alpha$-, and PR domaincontaining 16-PRDM16-) and mitochondrial function (UCP-1). Those mechanisms were also shown to occur in 3T3-L1 adipocytes subjected to inflammatory conditions ${ }^{44}$ or exposed to palmitate. ${ }^{45}$

Finally, it is relevant to consider that the observed taPVAT modifications were not associated with alterations in aorta remodeling, i.e. changes in the aorta wall thickness, inflammation, i.e. TNF- $\alpha$ expression, and determinants of superoxide anion and nitric oxide availability. The latter is suggested by the absence of changes in both aorta NOX2 catalytic subunit gp91, and the phosphorylation of eNOS. These results agree with the concept that some aspects of the vascular pathology induced by high-fat diets are due to the development of dysfunctional PVAT. ${ }^{41}$

It is important to note that for all parameters studied (-)-epicatechin had non-significant effects in mice fed the control diet. This absence of effects suggests that (-)-epicatechin (as well as other related flavonoids) generally mitigates 
A

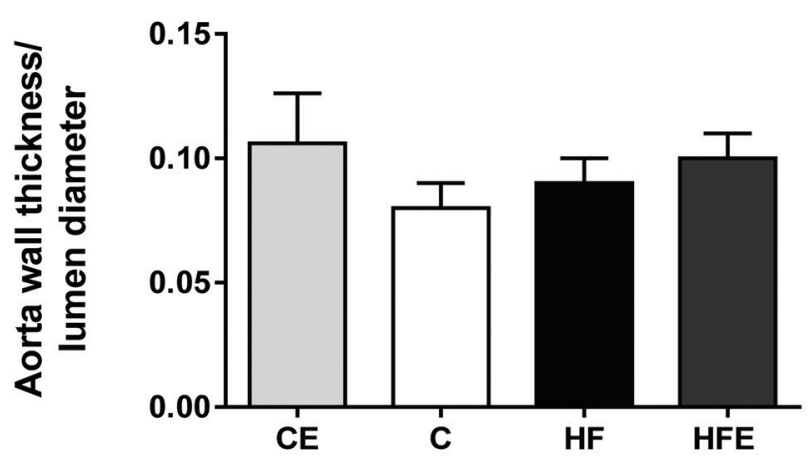

C
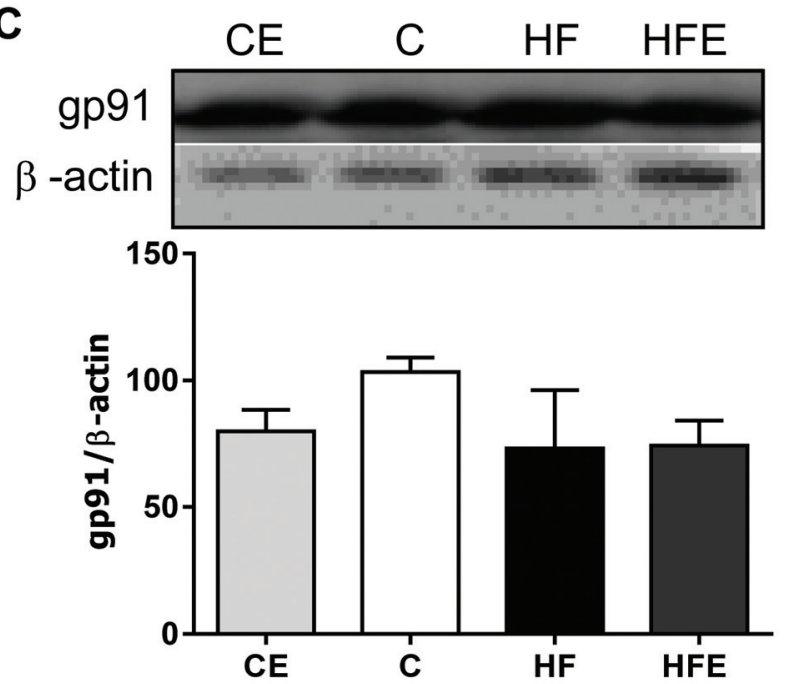

B

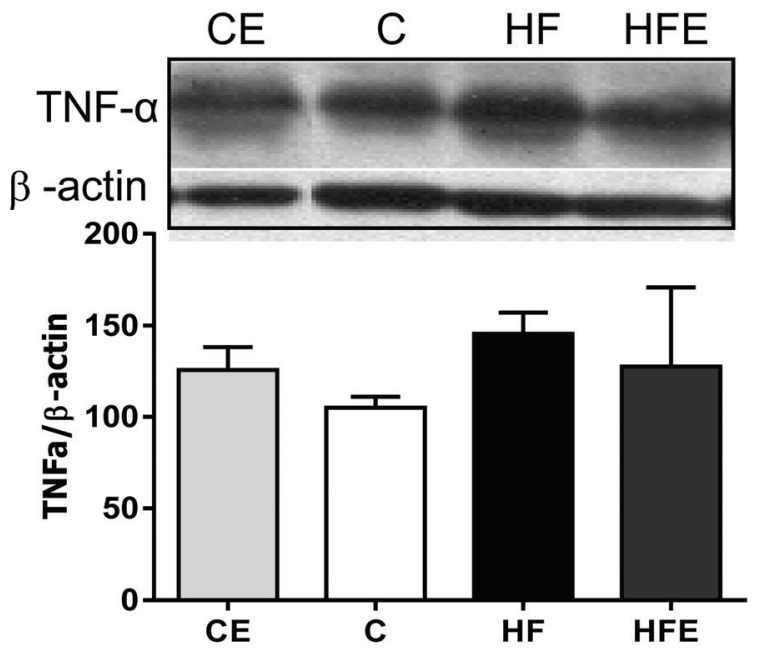

D
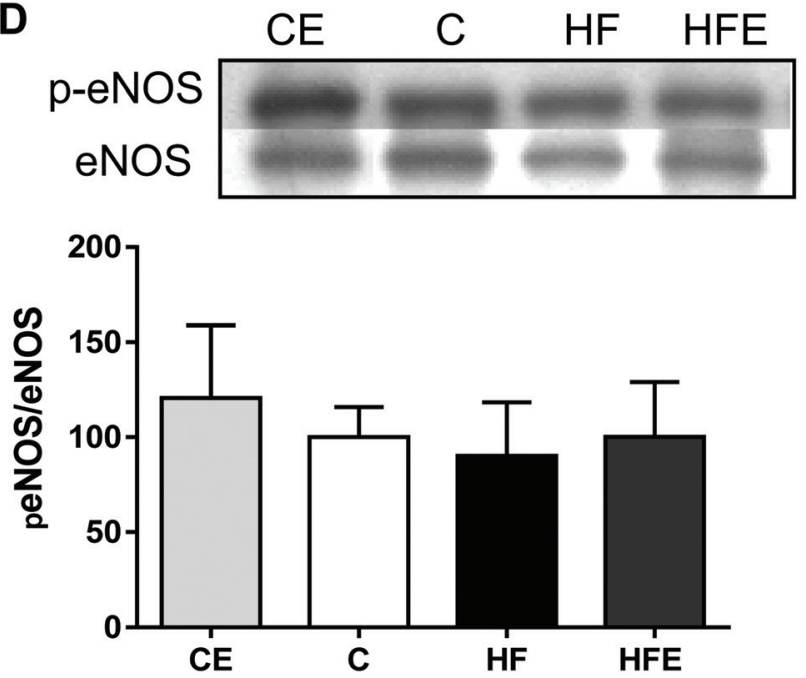

Fig. 7 Aorta morphometry and expression of TNF- $\alpha$, and gp91, and eNOS phosphorylation levels in the aorta. Wall thickness/lumen diameter (A), expression of TNF- $\alpha(B)$, and gp91 (C), and peNOS/eNOS ratio (D) in the aorta from CE, C, HF and HFE. $\beta$-Actin was used as the loading control. Results are expressed as means $\pm \operatorname{SEM}(n=5)$.

deviations related to pathological conditions, such those triggered by the high-fat diet consumption. ${ }^{16}$

In summary, (-)-epicatechin provided protecting effects in mice fed a high-fat diet in terms of fat metabolism. One of the most significant effects was the prevention of taPVAT acquisition of WAT features affording a crucial strategy to maintain a healthy vasculature. Other positive actions of (-)-epicatechin were observed reverting metabolic changes triggered by excessive fat consumption. These observations make (-)-epicatechin a valuable bioactive protecting from slow and silent development of metabolic diseases.

\section{Abbreviations}

AT Adipose tissue

BAT Brown adipose tissue
eNOS Endothelial nitric oxide synthase

eWAT Epididymal white adipose tissue

mWAT Mesenteric white adipose tissue

NOX2 NADPH-oxidase 2

iNOS Inducible nitric oxide synthase

pWAT Perirenal white adipose tissue

PVAT Perivascular adipose tissue

rWAT Retroperitoneal white adipose tissue

taPVAT Thoracic aorta perivascular adipose tissue

TNF- $\alpha$ Tumor necrosis factor

UCP-1 Uncoupling-protein 1

WAT White adipose tissue

\section{Conflicts of interest}

The authors declare no conflict of interest. 


\section{Acknowledgements}

The authors thank Dr Jorge E. Toblli from Laboratorio de Medicina Experimental, Hospital Alemán, Buenos Aires, Argentina for the assistance in histology and immunohistochemistry. This work was supported by grants from the University of Buenos Aires 20020170100586BA (MG), 20020160100132BA (CF), PIP-CONICET11220170100585CO (MG), and ANPCYT-PICT2018-03052 (MG).

\section{References}

1 A. R. Saltiel and J. M. Olefsky, Inflammatory mechanisms linking obesity and metabolic disease, J. Clin. Invest., 2017, 127, 1-4.

2 W. D. van Marken Lichtenbelt, J. W. Vanhommerig, N. M. Smulders, J. M. Drossaerts, G. J. Kemerink, N. D. Bouvy, P. Schrauwen and G. J. Teule, Cold-activated brown adipose tissue in healthy men, N. Engl. J. Med., 2009, 360, 1500-1508.

3 A. M. Cypess, S. Lehman, G. Williams, I. Tal, D. Rodman, A. B. Goldfine, F. C. Kuo, E. L. Palmer, Y. H. Tseng, A. Doria, G. M. Kolodny and C. R. Kahn, Identification and importance of brown adipose tissue in adult humans, $N$. Engl. J. Med., 2009, 360, 1509-1517.

4 T. P. Fitzgibbons, S. Kogan, M. Aouadi, G. M. Hendricks, J. Straubhaar and M. P. Czech, Similarity of mouse perivascular and brown adipose tissues and their resistance to diet-induced inflammation, Am. J. Physiol.: Heart Circ. Physiol., 2011, 301, H1425-H1437.

5 Y.-H. Lee, E. P. Mottillo and J. G. Granneman, Adipose tissue plasticity from WAT to BAT and in between, Biochim. Biophys. Acta, 2014, 1842, 358-369.

6 L. Badimon and J. Cubedo, Adipose tissue depots and inflammation: effects on plasticity and resident mesenchymal stem cell function, Cardiovasc. Res., 2017, 113, 10641073.

7 F. Perez-Vizcaino and C. G. Fraga, Research trends in flavonoids and health, Arch. Biochem. Biophys., 2018, 646, 107112.

8 X. Zhang, X. Li, H. Fang, F. Guo, F. Li, A. Chen and S. Huang, Flavonoids as inducers of white adipose tissue browning and thermogenesis: signalling pathways and molecular triggers, Nutr. Metab., 2019, 16, 47.

9 M. C. Litterio, G. Jaggers, G. Sagdicoglu Celep, A. M. Adamo, M. A. Costa, P. I. Oteiza, C. G. Fraga and M. Galleano, Blood pressure-lowering effect of dietary (-)-epicatechin administration in L-NAME-treated rats is associated with restored nitric oxide levels, Free Radical Biol. Med., 2012, 53, 1894-1902.

10 M. C. Litterio, M. A. Vazquez Prieto, A. M. Adamo, R. Elesgaray, P. I. Oteiza, M. Galleano and C. G. Fraga, (-)-Epicatechin reduces blood pressure increase in highfructose-fed rats: effects on the determinants of nitric oxide bioavailability, J. Nutr. Biochem., 2015, 26, 745-751.
11 P. D. Prince, C. R. Lanzi, J. E. Toblli, R. Elesgaray, P. I. Oteiza, C. G. Fraga and M. Galleano, Dietary ()-epicatechin mitigates oxidative stress, NO metabolism alterations, and inflammation in renal cortex from fructose-fed rats, Free Radical Biol. Med., 2016, 90, 35-46.

12 P. D. Prince, C. G. Fraga and M. Galleano, (-)-Epicatechin administration protects kidneys against modifications induced by short-term L-NAME treatment in rats, Food Funct., 2020, 11, 318-327.

13 V. Calabró, B. Piotrkowski, L. Fischerman, M. A. Vazquez Prieto, M. Galleano and C. G. Fraga, Modifications in nitric oxide and superoxide anion metabolism induced by fructose overload in rat heart are prevented by (-)-epicatechin, Food Funct., 2016, 7, 1876-1883.

14 B. Piotrkowski, V. Calabró, M. Galleano and C. G. Fraga, (-)-Epicatechin prevents alterations in the metabolism of superoxide anion and nitric oxide in the hearts of L-NAMEtreated rats, Food Funct., 2015, 6, 155-161.

15 E. Cremonini, A. Bettaieb, F. G. Haj, C. G. Fraga and P. I. Oteiza, (-)-Epicatechin improves insulin sensitivity in high fat diet-fed mice, Arch. Biochem. Biophys., 2016, 599, 13-21.

16 E. Cremonini, Z. Wang, A. Bettaieb, A. M. Adamo, E. Daveri, D. A. Mills, K. M. Kalanetra, F. G. Haj, S. Karakas and P. I. Oteiza, (-)-Epicatechin protects the intestinal barrier from high fat diet-induced permeabilization: Implications for steatosis and insulin resistance, Redox Biol., 2018, 14, 588-599.

17 A. Bettaieb, E. Cremonini, H. Kang, J. Kang, F. G. Haj and P. I. Oteiza, Anti-inflammatory actions of (-)-epicatechin in the adipose tissue of obese mice, Int. J. Biochem. Cell Biol., 2016, 81, 383-392.

18 A. Bettaieb, M. A. Vazquez Prieto, C. Rodriguez Lanzi, R. M. Miatello, F. G. Haj, C. G. Fraga and P. I. Oteiza, (-)-Epicatechin mitigates high-fructose-associated insulin resistance by modulating redox signaling and endoplasmic reticulum stress, Free Radical Biol. Med., 2014, 72, 247-256.

19 M. Gómez-Guzmán, R. Jiménez, M. Sánchez, M. J. Zarzuelo, P. Galindo, A. M. Quintela, R. LópezSepúlveda, M. Romero, J. Tamargo, F. Vargas, F. PérezVizcaíno and J. Duarte, Epicatechin lowers blood pressure, restores endothelial function, and decreases oxidative stress and endothelin-1 and NADPH oxidase activity in DOCA-salt hypertension, Free Radical Biol. Med., 2012, 52, 70-79.

20 O. H. Lowry, N. J. Rosebrough, A. L. Farr and R. J. Randall, Protein measurement with the Folin phenol reagent, J. Biol. Chem., 1951, 193, 265-275.

21 L. V. Yuzeforych and S. I. Musiyenko, Wilson GL and Rachek LI, Mitochondrial DNA damage and dysfunction, and oxidative stress are associated with endoplasmic reticulum stress, protein degradation and apoptosis in high fat diet-induced insulin resistance mice, PLoS One, 2013, 8, e54059.

22 M. S. Westerterp-Plantenga, A. Nieuwenhuizen, D. Tomé, S. Soenen and K. R. Westerterp, Dietary protein, weight 
loss, and weight maintenance, Annu. Rev. Nutr., 2009, 29, 21-41.

23 A. Astrup, The role of dietary fat in the prevention and treatment of obesity. Efficacy and safety of low-fat diets, Int. J. Obes. Relat. Metab. Disord., 2001, 25, S46-S50.

24 T. Sano, S. Nagayasu, S. Suzuki, M. Iwashita, A. Yamashita, T. Shinjo, T. Sanui, A. Kushiyama, T. Kanematsu, T. Asano and F. Nishimura, Epicatechin downregulates adipose tissue CCL19 expression and thereby ameliorates dietinduced obesity and insulin resistance, Nutr., Metab. Cardiovasc. Dis., 2017, 27, 249-259.

25 H. Cheng, N. Xu, W. Zhao, J. Su, M. Liang, Z. Xie, X. Wu and Q. Li, (-)-Epicatechin regulates blood lipids and attenuates hepatic steatosis in rats fed high-fat diet, Mol. Nutr. Food Res., 2017, 61, 11.

26 O. Osborn and J. M. Olefsky, The cellular and signaling networks linking the immune system and metabolism in disease, Nat. Med., 2012, 18, 363-374.

27 A. L. Ghaben and P. E. Scherer, Adipogenesis and metabolic health, Nat. Rev. Mol. Cell Biol., 2019, 20, 242258.

28 E. Kulenkampff and C. Wolfrum, Proliferation of nutrition sensing preadipocytes upon short term HFD feeding, Adipocyte, 2019, 8, 16-25.

29 B. Meissburger, J. Ukropec, E. Roeder, N. Beaton, M. Geiger, D. Teupser, B. Civan, W. Langhans, P. P. Nawroth, D. Gasperikova, G. Rudofsky and C. Wolfrum, Adipogenesis and insulin sensitivity in obesity are regulated by retinoid-related orphan receptor gamma, EMBO Mol. Med., 2011, 3, 637-651.

30 J. Wu, P. Boström, L. M. Sparks, L. Ye, J. H. Choi, A.-H. Giang, M. Khandekar, K. A. Virtanen, P. Nuutila, G. Schaart, K. Huang, H. Tu, W. D. van Marken Lichtenbelt, J. Hoeks, S. Enerback, P. Schrauwen and B. C. Spiegelman, Beige adipocytes are a distinct type of thermogenic fat cell in mouse and human, Cell, 2012, 150, 366-376.

31 S. Cinti, The adipose organ, Prostaglandins, Leukotrienes Essent. Fatty Acids, 2005, 73, 9-15.

32 C. G. Fraga and P. I. Oteiza, Dietary flavonoids: Role of (-)-epicatechin and related procyanidins in cell signaling, Free Radical Biol. Med., 2011, 51, 813-823.

33 C. G. Fraga and P. I. Oteiza, Bioactives and their impact on human health, Mol. Aspects Med., 2018, 61, 1.

34 S. De Los Santos, L. A. Reyes-Castro, R. M. Coral-Vázquez, J. P. Méndez, M. Leal-García, E. Zambrano and P. Canto,
(-)-Epicatechin reduces adiposity in male offspring of obese rats, J. Dev. Origins Health Dis., 2020, 11, 37-43.

35 L. Chang, M. T. Garcia-Barrio and Y. E. Chen, Perivascular Adipose Tissue Regulates Vascular Function by Targeting Vascular Smooth Muscle Cells, Arterioscler., Thromb., Vasc. Biol., 2020, 40, 1094-1109.

36 G. Lastra and C. Manrique, Perivascular adipose tissue, inflammation and insulin resistance: link to vascular dysfunction and cardiovascular disease, Horm. Mol. Biol. Clin. Invest., 2015, 22, 19-26.

37 T. P. Fitzgibbons and M. P. Czech, Epicardial and perivascular adipose tissues and their influence on cardiovascular disease: basic mechanisms and clinical associations, J. Am. Heart Assoc., 2014, 3, e000582.

38 S. B. Withers, C. E. Bussey, S. N. Saxton, H. M. Melrose, A. E. Watkins and A. M. Heagerty, Mechanisms of adiponectin-associated perivascular function in vascular disease, Arterioscler., Thromb., Vasc. Biol., 2014, 34, 1637-1642.

39 M. Gollasch, Vasodilator signals from perivascular adipose tissue, Br. J. Pharmacol., 2012, 165, 633-642.

40 T. Szasz and R. C. Webb, Perivascular adipose tissue: more than just structural support, Clin. Sci., 2012, 122, 1-12.

41 N. Xia and H. Li, The role of perivascular adipose tissue in obesity-induced vascular dysfunction, $\mathrm{Br}$. J. Pharmacol., 2017, 174, 3425-3442.

42 G. Gutiérrez-Salmeán, P. Ortiz-Vilchis, C. M. Vacaseydel, L. Garduño-Siciliano, G. Chamorro-Cevallos, E. Meaney, S. Villafaña, F. Villarreal, G. Ceballos and I. RamírezSánchez, Effects of (-)-epicatechin on a diet-induced rat model of cardiometabolic risk factors, Eur. J. Pharmacol., 2014, 728, 24-30.

43 C. E. Varela, A. Rodriguez, M. Romero-Valdovinos, P. Mendoza-Lorenzo, C. Mansour, G. Ceballos, F. Villarreal and I. Ramírez-Sánchez, Browning effects of (-)-epicatechin on adipocytes and white adipose tissue, Eur. J. Pharmacol., 2017, 811, 48-59.

44 M. A. Vazquez-Prieto, A. Bettaieb, F. G. Haj, C. G. Fraga and P. I. Oteiza, (-)-Epicatechin prevents TNF $\alpha$-induced activation of signaling cascades involved in inflammation and insulin sensitivity in 3 T3-L1 adipocytes, Arch. Biochem. Biophys., 2012, 527, 113-118.

45 C. Rodriguez Lanzi, D. J. Perdicaro, M. S. Landa, A. Fontana, A. Antoniolli, R. M. Miatello, P. I. Oteiza and M. A. Vazquez-Prieto, Grape pomace extract induced beige cells in white adipose tissue from rats and in 3 T3-L1 adipocytes, J. Nutr. Biochem., 2018, 56, 224-233. 